\title{
Promoter hypermethylation profiling of distant breast cancer metastases
}

\author{
Willemijne A. M. E. Schrijver ${ }^{1}$ Laura S. Jiwa $^{1} \cdot$ Paul J. van Diest $^{1}$ • \\ Cathy B. Moelans ${ }^{1}$
}

Received: 5 January 2015/Accepted: 26 March 2015/Published online: 5 April 2015

(c) The Author(s) 2015. This article is published with open access at Springerlink.com

\begin{abstract}
Promoter hypermethylation of tumor suppressor genes seems to be an early event in breast carcinogenesis and is potentially reversible. This makes methylation a possible therapeutic target, a marker for treatment response and/or a prognostic factor. Methylation status of 40 tumor suppressor genes was compared between 53 primary breast tumors and their corresponding metastases to brain, lung, liver, or skin. In paired analyses, a significant decrease in methylation values was seen in distant metastases compared to their primaries in $21 / 40$ individual tumor suppressor genes. Furthermore, primary tumors that metastasized to the liver clustered together, in line with the finding that primary breast carcinomas that metastasized to the brain, skin, or lung, showed higher methylation values in up to $27.5 \%$ of tumor suppressor genes than primary carcinomas that metastasized to the liver. Conversion in methylation status of several genes from the primary tumor to the metastasis had prognostic value, and methylation status of some genes in the metastases predicted survival after onset of metastases.
\end{abstract}

Electronic supplementary material The online version of this article (doi:10.1007/s10549-015-3362-y) contains supplementary material, which is available to authorized users.

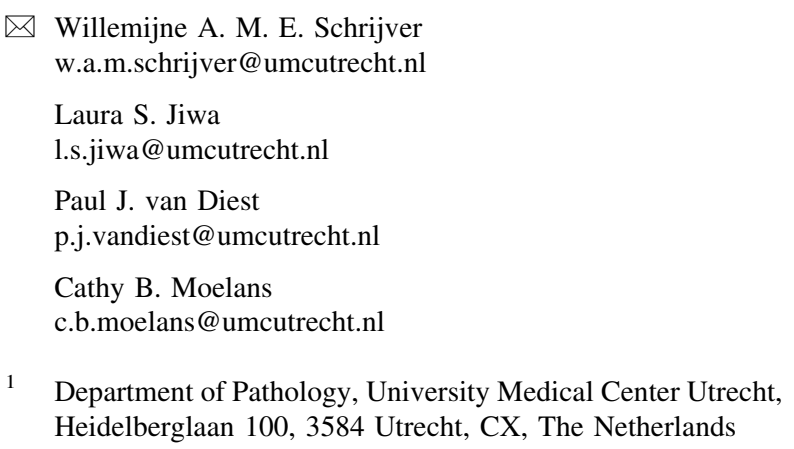

1 Department of Pathology, University Medical Center Utrecht, Heidelberglaan 100, 3584 Utrecht, CX, The Netherlands

Methylation levels for most of the analyzed tumor suppressor genes were lower in distant metastases compared to their primaries, pointing to the dynamic aspect of methylation of these tumor suppressor genes during cancer progression. Also, specific distant metastatic sites seem to show differences in methylation patterns, implying that hypermethylation profiles of the primaries may steer sitespecific metastatic spread. Lastly, methylation status of the metastases seems to have prognostic value. These promising findings warrant further validation in larger patient cohorts and more tumor suppressor genes.

Keywords Hypermethylation - Tumor suppressor genes · Breast cancer - Distant metastases - MLPA

\begin{tabular}{|c|c|}
\hline \multicolumn{2}{|c|}{ Abbreviations } \\
\hline MS-MLPA & $\begin{array}{l}\text { Methylation-specific multiplex ligand- } \\
\text { dependent probe amplification }\end{array}$ \\
\hline DNMT & DNA methyl-transferase inhibitors \\
\hline HDAC & Histone deacetylase inhibitors \\
\hline CMI & Cumulative methylation index \\
\hline $\mathrm{ER} \alpha$ & Estrogen receptor alpha \\
\hline PR & Progesterone receptor \\
\hline HER2 & Human epidermal growth factor receptor 2 \\
\hline MAI & Mitotic activity index \\
\hline $\mathrm{CpG}$ & Cytosine phosphate guanine \\
\hline QM-MSP & $\begin{array}{l}\text { Quantitative multiplex methylation-specific } \\
\text { PCR }\end{array}$ \\
\hline PCR & Polymerase chain reaction \\
\hline DTC & Disseminated tumor cell \\
\hline FFPE & Formalin-fixed paraffin embedded \\
\hline $\mathrm{P}$ & Primary \\
\hline M & Metastasis \\
\hline TSS & Transcription start site \\
\hline $\mathrm{Bp}$ & Base pairs \\
\hline
\end{tabular}




\section{Introduction}

With 1.7 million new cases causing 522,000 deaths worldwide per year, breast cancer is the leading cause of female cancer death [1]. Early detection, optimal surgery, and adjuvant therapy are the key strategies to improve prognosis. Although 5-year overall survival increased from $77 \%$ in the period $1978-1984$ to $82 \%$ in the period 1995-2003, about $16 \%$ of patients will develop distant metastases and eventually die of the disease [2]. Preferred site of distant metastases strongly depends on the subtype of breast cancer. Lobular-type breast cancer preferentially metastasizes to bone, GI tract and ovaries, triple negative breast cancer to liver and brain, and luminal breast cancer to the bone and skin, while well-circulated organs like the spleen and heart almost never harbor metastases [3-5]. This "organotropism" was first described by Paget et al. about a century ago as the "seed and soil" analogy, where tumors are supposed to have a "seminal influence" on the metastatic micro-environment, and thereby act together with the distant organ to effect tumor metastases [6]. The identity of these seminal influences remains elusive. Both genetic and epigenetic changes may play a role here. Epigenetic alterations are of pivotal interest since they cannot only influence tumor behavior but may also become important therapeutic targets as these processes are potentially reversible. Therapies that target DNA methylation (DNA methyl-transferase (DNMT) inhibitors) or histone modification (histone deacetylase (HDAC) inhibitors) already exist, but newer versions of these drugs need to be developed to improve future clinical management [7].

Which mechanisms underlie development of distant metastases remains a topic of debate. The two main but not necessarily mutually exclusive hypotheses are the linear and the parallel model of metastasis. According to the linear model, genetic modifications progressively accumulate in cancer cells of the primary tumor, whereby cells with advantageous mutations will survive and expand through clonal evolution [8]. If we translate this into epigenetic alterations such as promoter hypermethylation, one would expect that tumor suppressor genes in metastases show more methylation than primary carcinomas. An increase in methylation values during local tumor progression has already been shown $[9,10]$. In the parallel progression model, cancer cells disseminate early during tumor progression at a stage when the primary lesion is small. Disseminated cells then evolve independently of the primary tumor to form metastases. According to this latter model, one would expect different methylation patterns in primaries and their matched metastases.

Hypermethylation of tumor suppressor genes like $A P C$, RASSF 1A, and FEZ1/LZTS1 in primary breast cancer has been reported to correlate with development of distant metastases [11, 12]. However, little is known about the comparative methylation status of primary tumors and matched distant metastases, possibly related to the fact that metastatic material is rare. Rivenbark et al. compared the methylation status of CST6 in primary breast cancers to their lymph node metastases and showed that methylationdependent silencing occurred more frequently in the lymph node metastases, possibly reflecting progression-related epigenetic events according to the linear model for metastasis [13].

Here we report promoter hypermethylation profiling for 40 tumor suppressor genes by methylation-specific multiplex ligation-dependent probe amplification (MS-MLPA) in 53 primary breast carcinomas and their matched nonbone distant metastases (skin, brain, lung or liver). This study is part of a project where we study genotype and phenotype of distant breast cancer metastases [14-16]. Extensive knowledge of the hypermethylation status of tumor suppressor genes possibly involved in site-specific metastasis could lead to novel biomarkers predicting site of distant metastases and adjuvant targeted therapy strategies that could prevent such metastases from becoming clinically manifest.

\section{Materials and methods}

\section{Patients}

This study was performed on 53 formalin-fixed paraffin embedded (FFPE) samples of female primary breast carcinomas and 53 single corresponding metachronous nonbone distant metastases. The samples were selected randomly from an existing database entailing material from 300 patients from the departments of pathology of the University Medical Center Utrecht, the Meander Medical Center Amersfoort, the Deventer Hospital, the Rijnstate Hospital Arnhem, Tergooi Hospitals, the Academic Medical Center Amsterdam, the Radboud University Nijmegen Medical Center, the Canisius Wilhelmina Hospital Nijmegen, the Netherlands Cancer Institute Amsterdam, the Medical Center Alkmaar, the Medical Center Zaandam, the University Medical Center Groningen, the St. Antonius Hospital Nieuwegein, the Diakonessenhuis Utrecht, the Free University Medical Center Amsterdam, the Erasmus Medical Center Rotterdam, the Gelre hospital Apeldoorn, Isala clinics Zwolle, the Laboratory for Pathology Enschede, the Laboratory for Pathology Dordrecht, and the Laboratory for Pathology Foundation Sazinon Hoogeveen, all in The Netherlands.

This study was performed in accordance with the institutional medical ethical guidelines. The use of anonymous 
or coded left over material for scientific purposes is part of the standard treatment agreement with patients, and therefore, informed consent was not required according to Dutch law [17].

Molecular subtypes of breast tumors were assigned as follows: Luminal A (ER+/PR+, HER2-, low cellular proliferation), luminal B (ER+/PR+, HER2-, low cellular proliferation or $\mathrm{ER}+/ \mathrm{PR}+, \mathrm{HER} 2+$ ), triple negative or basal type (ER-/PR-, HER2-), and HER2 enriched (ER-/PR-, HER2+) as before [4].

To set methylation cut-off values, non-paired normal breast tissue $(n=25)$ was used from breast reduction specimens (mean age 39.4 years; $n=15$ ) and autopsy specimens (mean age 48.9 years; $n=10$ ), with no significant difference in age compared to breast cancer patients $(p=0.338)$. In addition, we analyzed normal nonpaired tissue from brain $(n=5)$, lung $(n=5)$, liver $(n=5)$, and skin $(n=5)$ derived from our normal tissue biobank to exclude that methylation values in distant metastases would be influenced by admixture of normal surrounding tissue, with again no significant difference in age (45.8 years) compared to patients with breast cancer $(p=0.111)$. The mean patient age at diagnosis was 52.8 years and $84 \%$ of patients presented with invasive ductal carcinoma. Follow-up ranged between sixteen and 315 months, and metastases were meanly diagnosed 55.4 months after the primary diagnosis. The localization of the metastases that were included was brain $(n=11)$, lung $(n=12)$, liver $(n=10)$, and skin $(n=20)$. Clinicopathological characteristics are shown in Table 1.

\section{DNA extraction}

Four-micrometer sections were cut from each FFPE tissue block and stained with haematoxylin and eosin (HE). The HE-section was used to guide macro-dissection for DNA extraction and to estimate tumor percentage. Only samples containing 80 per cent tumor load or higher (both primary tumor and metastasis) were selected. For proteinase K-based DNA extraction, five 5- $\mu \mathrm{m}$-thick slides were cut, and tumor areas were macro-dissected using a scalpel. Areas with necrosis, dense lymphocytic infiltrates, and preinvasive lesions were intentionally avoided. The DNA concentration and absorbance at 260 and $280 \mathrm{~nm}$ were measured with a spectrophotometer (Nanodrop ND-1000, Thermo Scientific Wilmington, USA).

\section{MS-MLPA}

MS-MLPA was performed according to the manufacturer's protocol using the SALSA MS-MLPA probemixes ME001C2 Tumor suppressor-1 and ME003-A1 Tumor suppressor3 "Online Resource Tables 1 and 2," each containing 15 internal control probes and in total 53 HhaI-sensitive probes against the following tumor suppressor genes: TP73, CASP8, VHL, RARB, MLHI (2 loci), RASSF1A (2 loci), FHIT, APC, ESR1, CDKN2A/B, DAPK1, KLLN, CD44, GSTP1, ATM, CADM1, CDKN1B, CHFR, BRCA1/ 2, CDH13, HICl, TIMP3 (2 loci), RDM2, RUNX3, HLTF (2 loci), SCGB3A1 (2 loci), ID4 (2 loci), TWISTI, SFR4 (2 loci), DLC1 (2 loci), SFR5 (2 loci), BNI3, H2AFX (2 loci), CCND2 (2 loci), CACNA1G, TGIF1, BCL2, and CAC$N A 1 A$. Since MS-MLPA is based on the methylation-sensitive restriction enzyme $\mathrm{HhaI}$, the choice of $\mathrm{CpG}$ site to be evaluated within the promoter region is highly dependent on the presence of the GCGC restriction site and not so much based on correlation to expression in literature.

At least 50 ng of DNA was used in each MS-MLPA reaction. DNA concentration control fragments, present in each MS-MLPA mix, were evaluated to check for sufficient DNA quantity. All reactions were performed according to the manufacturer's instructions in a Veriti 96 Well Thermo Cycler (Applied Biosystems). A water sample, a $100 \%$ methylated (MCF-7 M.SssI methyl-transferase treated) control, and a negative control (human sperm DNA) were taken along in every MLPA run. Fragment separation was done by capillary electrophoresis on an ABI-3730 capillary sequencer (Applied Biosystems). Peak patterns derived by Genescan Analysis were evaluated using Genemapper (version 4.1) and Coffalyser.net software (version 9.4, MRC-Holland, Amsterdam, The Netherlands). The cumulative methylation index (CMI) was calculated as the sum of all quantitative methylation values per tumor. Raw methylation percentages of all genes were depicted in "Online Resource Table 7."

\section{Correlation between mRNA expression and promoter methylation by TCGA}

To correlate methylation of the investigated tumor suppressor genes to mRNA expression, we used The Cancer Genome Atlas (https://tcga-data.nci.nih.gov/tcga/).

TCGA Breast Invasive Carcinoma mRNA Expression z-Scores (RNA Seq V2 RSEM) data $(n=1038)$ were downloaded via The cBioPortal for Cancer Genomics [18, 19]. Illumina Infinium Human DNA Methylation 27 level 3 data (calculated beta values $(\mathrm{M} / \mathrm{M}+\mathrm{U})$, gene symbols, chromosomes, and genomic coordinates) were downloaded via TCGA Data Portal $(n=313)$.

Statistical analyses were performed on data of all available $\mathrm{CpG}$ sites of the TCGA database compared to the $\mathrm{CpG}$ sites used for MS-MLPA.

\section{Statistics}

Unsupervised hierarchical clustering of log-transformed quantitative methylation values was performed using non- 
Table 1 Clinicopathological characteristics of the metastatic breast cancer patients $(n=53)$ analyzed for methylation status of 40 tumor suppressor genes with MS-MLPA

\begin{tabular}{|c|c|c|c|}
\hline Feature & Grouping & $N$ or value & $\%$ \\
\hline \multirow[t]{2}{*}{ Age at diagnosis (in years) } & Mean & 52.8 & - \\
\hline & Range & $27-88$ & - \\
\hline \multirow[t]{4}{*}{ Tumor size (in $\mathrm{cm}$ ) } & $\leq 2$ & 16 & 30 \\
\hline & $>2$ and $\leq 5$ & 26 & 49 \\
\hline & $>5$ & 6 & 11 \\
\hline & Not available & 5 & 10 \\
\hline \multirow[t]{4}{*}{ Histologic type } & Invasive ductal & 45 & 84 \\
\hline & Invasive lobular & 4 & 8 \\
\hline & Metaplastic & 3 & 6 \\
\hline & Micropapillary & 1 & 2 \\
\hline \multirow[t]{3}{*}{ Histologic grade (Bloom \& Richardson) } & I & 1 & 2 \\
\hline & II & 12 & 22 \\
\hline & III & 40 & 76 \\
\hline \multirow[t]{4}{*}{ MAI (per $2 \mathrm{~mm}^{2}$ ) } & Mean & 24.8 & 26 \\
\hline & Range & $0-86$ & 74 \\
\hline & $\leq 12$ & 14 & - \\
\hline & $\geq 13$ & 39 & - \\
\hline \multirow[t]{3}{*}{ Lymph node status } & Positive & 25 & 47 \\
\hline & Negative & 24 & 45 \\
\hline & Not available & 4 & 8 \\
\hline \multirow[t]{4}{*}{ Site of distant metastasis } & Brain & 11 & 21 \\
\hline & Lung & 12 & 22 \\
\hline & Liver & 10 & 19 \\
\hline & Skin & 20 & 38 \\
\hline \multirow[t]{4}{*}{ Molecular subtype } & Luminal A & 11 & 21 \\
\hline & Luminal B & 28 & 53 \\
\hline & Triple negative & 12 & 22 \\
\hline & HER2 enriched & 2 & 4 \\
\hline \multirow[t]{2}{*}{ Follow-up in months } & Mean & 94 & - \\
\hline & Range & $16-315$ & - \\
\hline \multirow[t]{2}{*}{ Time between diagnosis of primary and metastasis (in months) } & Mean & 55.4 & - \\
\hline & Range & $0.4-180.8$ & - \\
\hline \multirow[t]{2}{*}{ Time between diagnosis of metastasis and death (in months) } & Mean & 26.6 & - \\
\hline & Range & $2.0-177.7$ & - \\
\hline \multirow{5}{*}{$\begin{array}{l}\text { Treatment before resection of metastasis (adjuvant to } \\
\text { surgery of primary breast tumor) }\end{array}$} & Chemotherapy & 19 & 36 \\
\hline & Hormonal therapy & 17 & 32 \\
\hline & Radiotherapy & 26 & 49 \\
\hline & $\begin{array}{l}\text { Combination of chemo-, hormonal } \\
\text { and/or radiotherapy }\end{array}$ & 22 & 42 \\
\hline & Not available & 13 & 25 \\
\hline \multicolumn{4}{|l|}{ ER status ${ }^{\mathrm{a}}$} \\
\hline \multirow[t]{2}{*}{ Primary } & + & 36 & 68 \\
\hline & - & 17 & 32 \\
\hline \multirow[t]{2}{*}{ Metastasis } & + & 35 & 66 \\
\hline & - & 18 & 34 \\
\hline \multicolumn{4}{|l|}{ PR status ${ }^{a}$} \\
\hline \multirow[t]{2}{*}{ Primary } & + & 33 & 62 \\
\hline & - & 20 & 38 \\
\hline
\end{tabular}


Table 1 continued

\begin{tabular}{|c|c|c|c|}
\hline Feature & Grouping & $N$ or value & $\%$ \\
\hline \multirow[t]{2}{*}{ Metastasis } & + & 22 & 42 \\
\hline & - & 31 & 58 \\
\hline \multicolumn{4}{|l|}{ HER2-status ${ }^{\mathrm{b}}$} \\
\hline \multirow[t]{4}{*}{ Primary } & 0 & 41 & 77 \\
\hline & $1+$ & 4 & 8 \\
\hline & $2+$ & 1 & 2 \\
\hline & $3+$ & 7 & 13 \\
\hline \multirow[t]{4}{*}{ Metastasis } & 0 & 38 & 72 \\
\hline & $1+$ & 5 & 9 \\
\hline & $2+$ & 4 & 8 \\
\hline & $3+$ & 6 & 11 \\
\hline
\end{tabular}

MAI mitotic activity index

${ }^{a}$ According to $10 \%$ threshold for positivity

b According to DAKO-scoring system

parametric Spearman correlation with R software (version 3.0.1), including all cases that were tested with both MLPA probemixes. Statistical analysis was executed on absolute methylation percentages as well as on dichotomized values; the latter were determined by ROC curve analyses of methylation values in normal breast tissue compared to primary breast tumor tissue. The Kolmogorov-Smirnov test and Shapiro-Wilk test were used to test for normality of the distributions. Primary tumors and their paired metastases were compared per gene using the Wilcoxon signed-rank test. Non-paired analyses on patient differences and clinicopathological characteristics were computed using the Mann-Whitney test. The dichotomized values were analyzed using McNemars test or Chi square test. Two-sided $p$ values $<0.05$ were considered to be statistically significant. Correction for multiple comparisons was performed by the Bonferroni-Holm approach. Analysis of prognosis was performed using Kaplan-Meier survival curves/log-rank test for univariate analyses and Cox proportional hazard analysis for multivariate models (entry and remove limits 0.05 ), calculating hazard ratios (HR) with $95 \%$ confidence intervals (CI). TCGA mRNA z-scores were compared to percentages of DNA methylation by Pearson's $r$ correlation.

To evaluate whether site of distant metastasis is determined by specific methylation patterns of the primary tumor or rather by inherent molecular subtype, we performed logistic regression comparing the different metastatic sites one by one with quantitative methylation status of individual genes and molecular subtype as variables in the model.

To evaluate whether adjuvant systemic treatment may influence conversion from low methylation in the primary to high methylation in the distant metastasis (or vice versa), we grouped patients according to conversion per individual gene and performed logistic regression for each individual gene including adjuvant chemotherapy (yes or no) and adjuvant hormonal therapy (yes or no) as variables in the model.

All statistical calculations were done with IBM SPSS Statistics 21.

\section{Results}

\section{Normal versus tumor tissue}

Appropriate cut-offs to dichotomize methylation values of tumor suppressor genes, derived from ROC curve analysis of MS-MLPA values in normal breast versus primary breast tumor tissue, varied between 0.5 and $22.75 \%$ for the 40 genes (53 loci) (Online Resource Table 8).

Although we only included samples of breast cancer metastases that contained 80 percent tumor load or higher, we wanted to further exclude that differences between primaries and metastases were due to the admixture of tumor micro-environment at distant sites. 17/40 genes showed significantly higher methylation values in normal lung, brain, or liver than in normal breast (Online Resource Table 3; Fig. 1a shows CASP8 as an example). Also the CMI values of normal liver and brain tissue were significantly higher than the CMI of normal lung, skin, and breast tissue (Fig. 1b).

Unsupervised hierarchical clustering of the quantitative methylation values of primary breast tumors, paired distant metastases, and normal tissues is shown in Fig. 2. Normal liver and brain tissue seems to cluster together due to hypermethylation of some genes $(A P C, C D K N 2 B, C C N D 2$ 


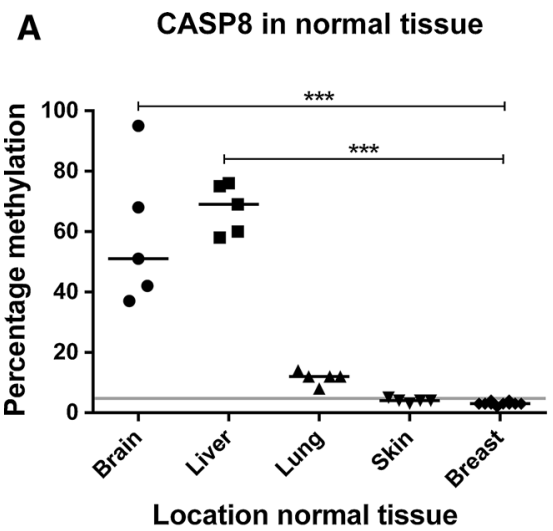

Fig. 1 Differences in quantitative methylation percentages of CASP8 a and the CMI b by MS-MLPA between various normal tissues. $N=30$ (brain $n=5$, liver $n=5$, lung $n=5$, skin $n=5$, and breast $n=10)$. Small horizontal lines depict the median per group. The gray

both loci, RASSF1A both loci and CASP8) as already mentioned above, and normal breast, lung, and skin tissue showed a related pattern.

\section{Primary tumor versus metastasis}

Using quantitative methylation values, $52.5 \%$ (21/40) of genes were significantly less methylated in the metastases compared to their paired primary tumors : PRDM2 $(p=0.036), R A R B-2(p=0.003), H L T F-2(p=0.013)$, H2AFX-1 $(p=0.001)$, CACNAIG $(p=0.000)$, TGIF1 $(p=0.029)$, TIMP3-1 $(p=0.046)$, TP73 $(p=0.019)$, FHIT $(p=0.002), A P C(p=0.048), C D K N 2 A(p=0.002)$, CDKN2B $\quad(p=0.012), \quad$ PTEN $\quad(p=0.002), \quad C D 44$ $(p=0.011), A T M(p=0.000), C A D M 1(p=0.006), C H F R$ $(p=0.005), B R C A 2(p=0.001), H I C l(p=0.001)$, and $B R C A 1$ ( $p=0.002)$. After correction for multiple comparisons, $H 2 A F X-1, C A C N A 1 G, A T M, B R C A 2$, and HICl remained significant. CMI was not significantly different between primaries and metastases $(p=0.454)$. Figure $3 \mathrm{a}$ shows quantitative methylation values of $C A C N A 1 G$ in primary tumors and their distant metastases as an example.

Using dichotomized values, $55 \%$ (22/40) of the tested tumor suppressor genes, namely PRDM2 $(p=0.049), R A R B-1$ $(p=0.002)$, HLTF-2 $(p=0.031)$, TWISTI $(p=0.012)$, $H 2 A F X$ both loci $(p=0.002$ and $p=0.049), C A C N A 1 G$ $(p=0.013)$, TGIF1 $(p=0.002)$, TIMP3-3 $(p=0.013)$, TP73 $(p=0.007)$, FHIT $(p=0.001), C D K N 2 A(p=0.029)$, DAPK1 $(p=0.004), \operatorname{PTEN}(p=0.008), C D 44(p=0.000)$, $\operatorname{GSTPl}(p=0.013), \operatorname{ATM}(p=0.000), C A D M 1(p=0.000)$, CHFR $(p=0.031), B R C A 2(p=0.013), H I C l(p=0.016)$, and $B R C A l(p=0.000)$, were significantly less methylated in the metastases than in the primaries. After correction for multiple comparisons, FHIT, CD44, ATM, CADM1, and $B R C A l$ stayed significant.

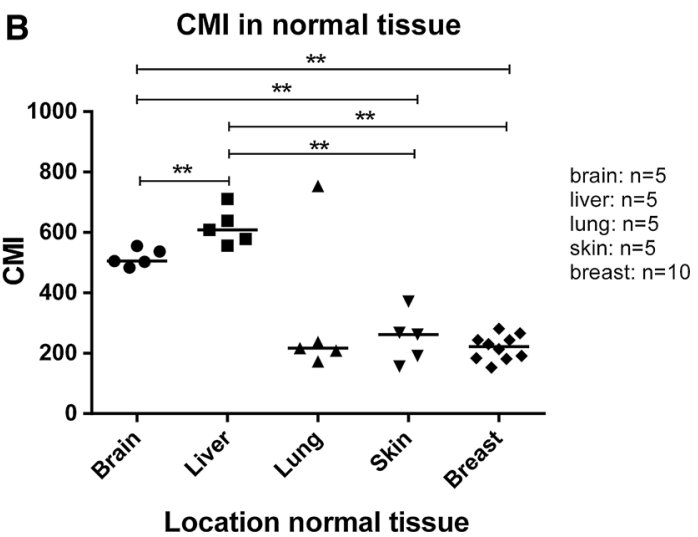

horizontal line depicts the cut-off for hypermethylation of CASP8 (4.5\%). Chromosome location CASP8: chr2 (202122754202152434), CpG site MS-MLPA probe: 202122649, \#bp from probe to TSS: 104 and from probe to ATG: 104

PRDM2, HLTF-2, H2AFX-1, CACNA1G, TGIF1, TP73, FHIT, CDKN2A, PTEN, CD44, ATM, CADM1, CHFR, $B R C A 2, H I C l$, and $B R C A l$ were significant in both quantitative and dichotomized analyses. Of these, PRDM2, H2AFX-1, TGIF1, TP73, CDKN2A, and CD44 were more methylated in normal brain and/or liver tissues than in normal breast, which indicates that the generally lower methylation values in the distant metastases must be tumor cell specific and excludes the potential admixture of cells from the distant microenvironment being a confounder here.

When comparing primaries and metastases for all investigated tumor suppressor genes per individual patient, significantly less methylation was seen in the metastases compared to the primary tumor in $30.2 \%$ (16/53; quantitative) or $41.5 \%$ (22/53; dichotomized) of patients (20.8 and $28.3 \%$ after correction for multiple comparisons, respectively). Only $15.1 \%$ (8/53; quantitative) or $3.8 \%$ (2/ 53; dichotomized) of patients showed significantly more methylation in the metastasis compared to the primary tumor (3.8 or $1.9 \%$, respectively, if corrected for multiple comparisons). These higher methylation values cannot be explained by admixture of normal adjacent tissue in the metastases, since none of these patients had a metastasis in brain or liver, where high methylation values are found in normal tissue.

In cluster analysis (Fig. 2), 32/53 pairs of primaries and metastases clustered directly and another $9 / 53$ pairs almost directly (within three positions), indicating that methylation patterns of the tested tumor suppressor genes show high patient specificity.

\section{Molecular subtype}

HER2 enriched tumors were excluded from statistical analyses because of the small number. Triple negative 


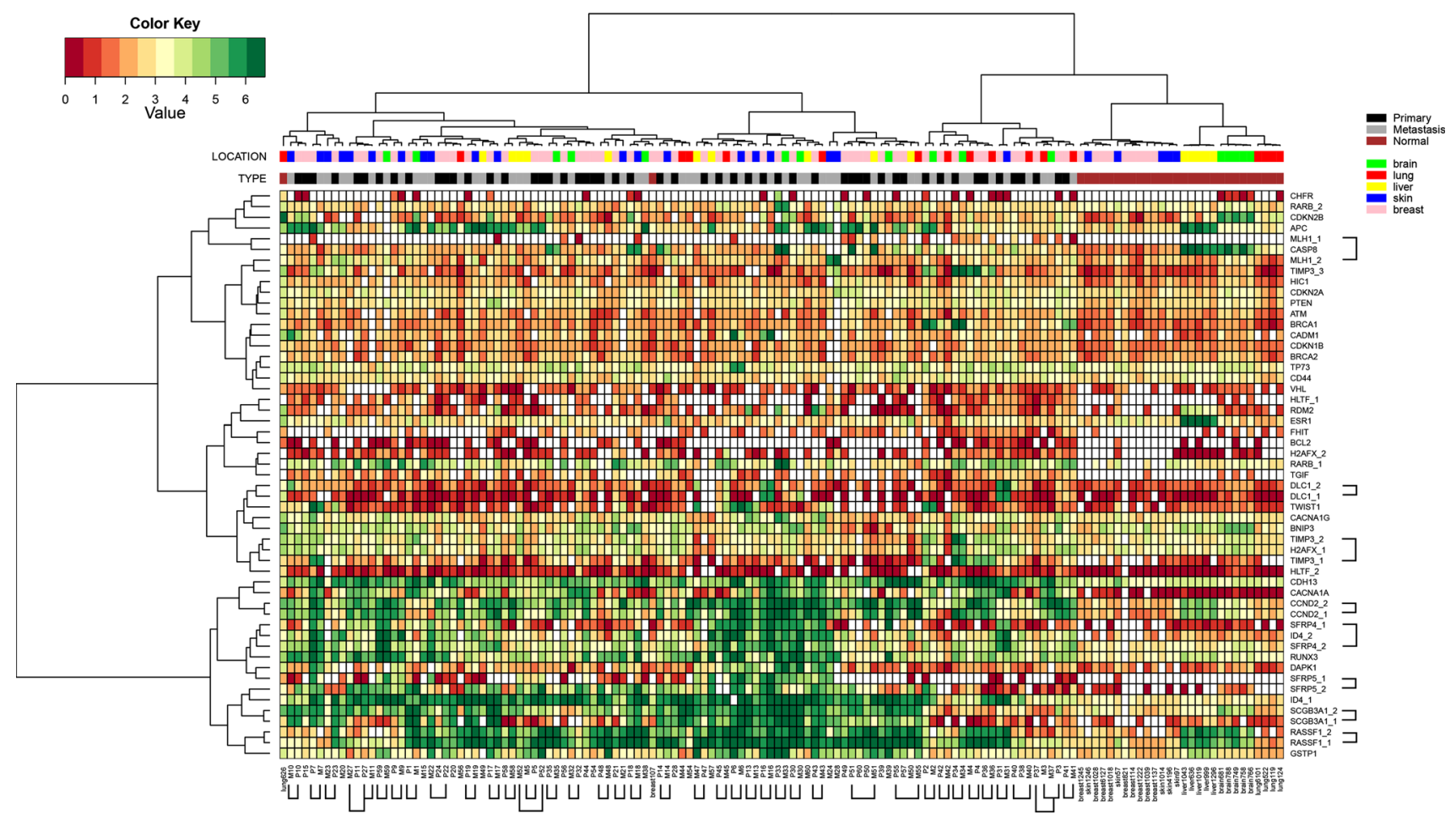

Fig. 2 Unsupervised hierarchical clustering analysis of log-transformed quantitative methylation percentages of 40 tumor suppressor genes (53 loci) in 53 primary breast tumors, 53 paired distant

tumors tended to cluster together, but the difference between luminal $\mathrm{A}$ and $\mathrm{B}$ was less distinct (Fig. 4).

PRDM2, RARB, CACNAIG (Fig. 3b), SFRP4-2, H2AFX, CACNAIA, TIMP3-1/2, and DLC1-1 showed significantly less methylation in luminal A primary tumors compared to luminal $\mathrm{B}$ and/or triple negative primary tumors. Less methylation of SCGB3Al was seen in triplenegative tumors compared to the other subtypes. Further, more methylation of ID4-2 was seen in luminal B tumors compared to the other subtypes. When corrected for metastatic site, these effects disappeared (Fig. 3c), indicating that although subgroups were small, molecular subtype is not a significant determinant of dissemination site in this group (Online Resource Table 4). No differences were seen between the CMI of the different molecular subtypes ( $p=0.199)$ (Online Resource Table 5).

Concerning receptor status, $35 \%$ (14/40; quantitative) or $25 \%$ (10/40; dichotomized) of the tumor suppressor genes showed significantly higher methylation values in ER-positive tumors compared to ER-negative tumors. After correction for multiple comparisons, $5 \%$ of the tumor suppressor genes remained significant for both data types: SCGB3A1 (both loci), ID4-1, SFRP5-2, H2AFX-1, and FHIT.

In PR-positive tumors, this phenomenon was less distinct: 17.5 or $25 \%$ of genes (quantitative or dichotomized metastases, and 30 normal tissues (breast $n=10$, brain $n=5$, lung $n=5$, liver $n=5$, and skin $n=5$ ). The sidebars depict location of tissue and type (primary, metastasis, or normal tissue)

respectively) showed higher methylation values, but no significance remained after multiple comparisons correction. Further, in HER2-positive tumors more methylation was seen in $2.5 \%$ (quantitative) or $7.5 \%$ (dichotomized) of tumor suppressor genes, but again no significance remained when corrected for multiple comparisons.

\section{Metastatic site}

The following genes were significantly more methylated in primary tumors metastasizing to brain, lung, or skin, than to liver: PRDM2 (quantitative and dichotomized), RARB-1 (quantitative and dichotomized), HLTF-1 (quantitative), ID4-2 (quantitative), TWIST1 (quantitative and dichotomized), SFRP4-2 (quantitative an dichotomized), $D L C 1$ (both loci; quantitative), $H 2 A F X-2$ (quantitative and dichotomized), CACNAIG (quantitative and dichotomized) (Fig. 3d), CACNAIA (quantitative), and TIMP3 (all three loci; quantitative, $-\mathrm{b}$; dichotomized). Also in the heatmap (Fig. 4), a distinct cluster was formed by primary breast tumors that metastasized to liver.

When corrected for molecular subtype by logistic regression, the largest differences in methylation of individual genes were seen between liver and skin (skin being more methylated), and also the CMI was significantly different here $(p=0.039)$. Figure $3 \mathrm{e}$ shows significantly 

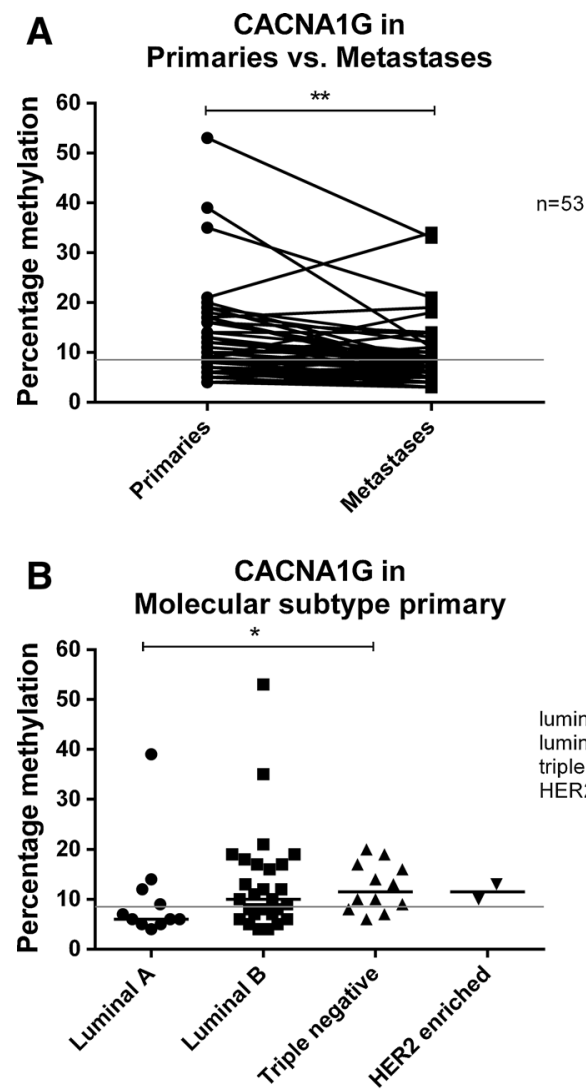

D CACNA1G in Location of dissemination

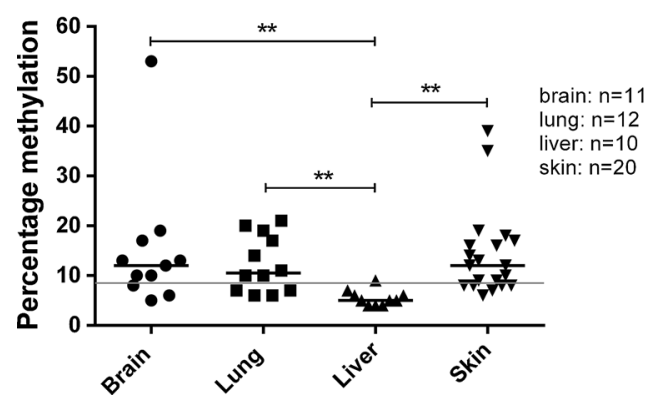

Fig. 3 Quantitative methylation percentages of CACNA1G by MSMLPA in primary breast tumors and their corresponding distant metastases (a). Methylation percentages in the primary tumor, divided per molecular subtype (b) and corrected for dissemination localization (brain) (c) are shown thereunder. At the bottom, methylation percentages in the primary tumor, divided per dissemination location

more methylation of CACNA1G in brain, lung, and skin compared to liver (quantitative data) as an example.

\section{Association with clinicopathological characteristics}

"Online Resource Table 5" shows the association between methylation in the primary tumor and classical clinicopathological characteristics. A higher CMI (quantitative values) significantly correlated with higher MAI

\section{CACNA1G in Subtype corrected for location (brain)}

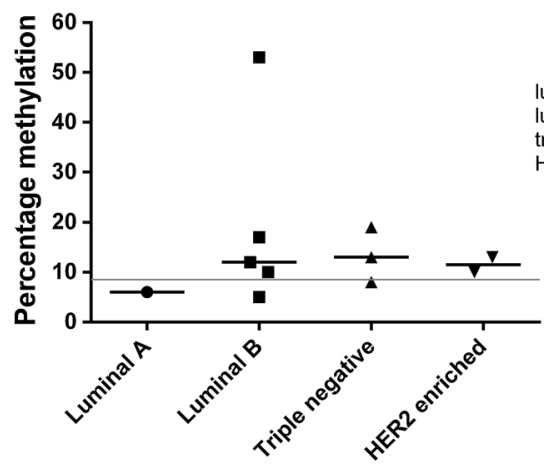

E CACNA1G in Location corrected for subtype (luminal B)

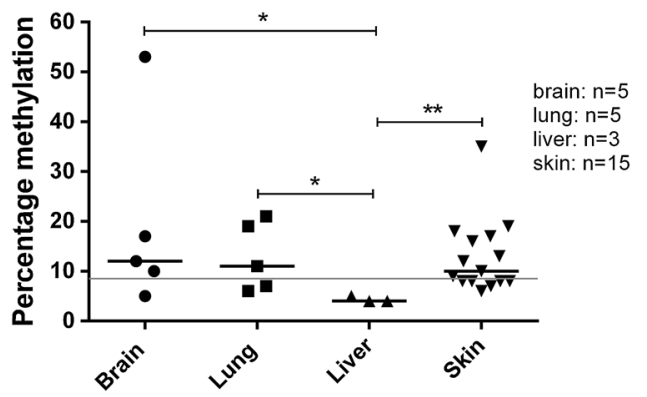

(d) and corrected for molecular subtype (luminal B) (e), are presented. Small horizontal lines depict the median per group. The gray horizontal line depicts the cut-off for hypermethylation $(8.5 \%)$. Chromosome location CACNA1G: chr17:48638429-48704832, CpG site MS-MLPA probe: 48638728 , \#bp from probe to TSS: -300 and from probe to ATG: 92

$(p=0.040)$, although there was no association to lymph node status, localization of metastases, and molecular subtype. More aggressive tumor characteristics like higher grade and MAI showed a tendency to higher methylation values of individual genes.

Logistic regression for methylation conversion between the primary cancers and their metastases did not show significance for chemotherapy or hormonal therapy for any of the genes, indicating that adjuvant systemic treatment is 


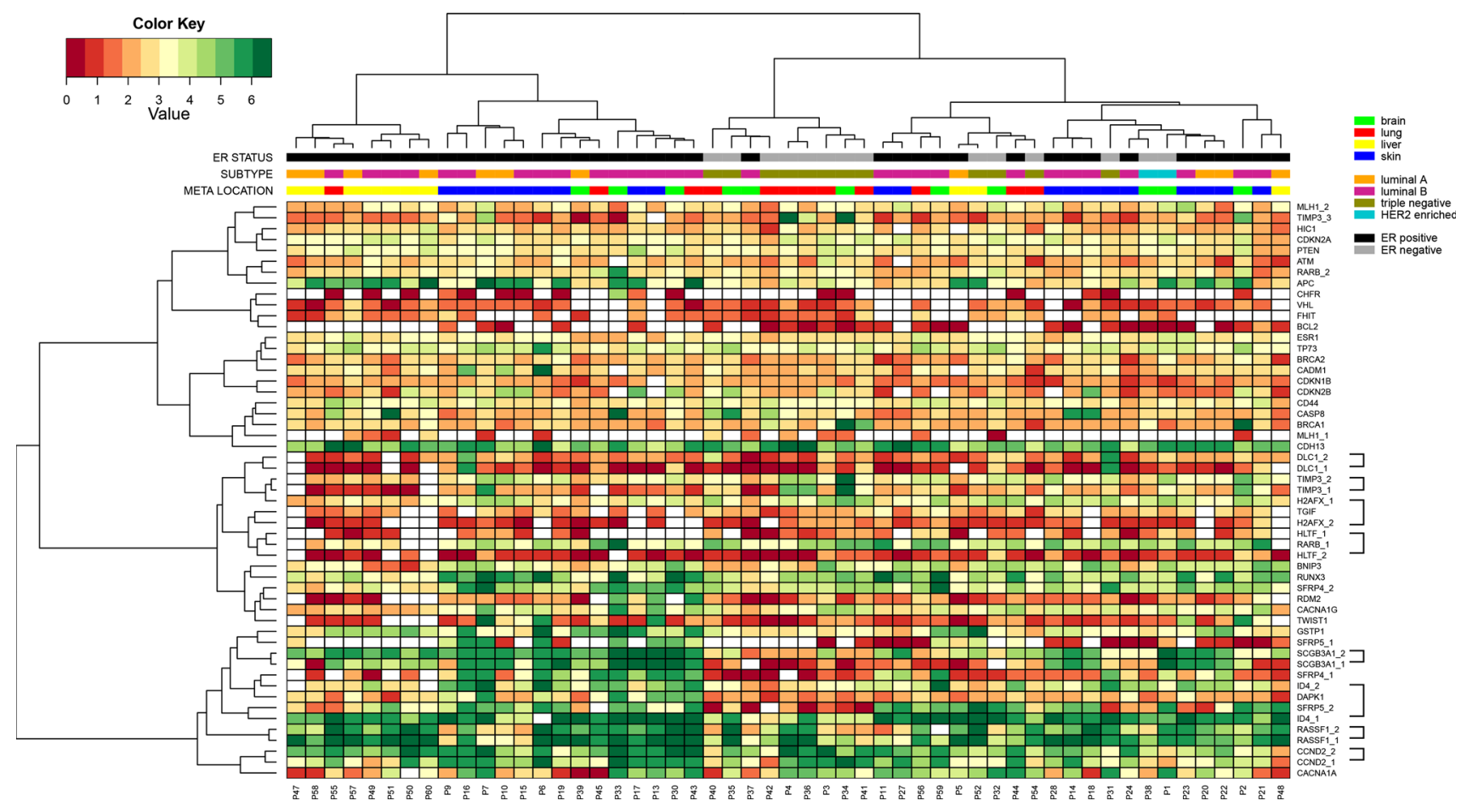

Fig. 4 Unsupervised hierarchical clustering analysis of log-transformed quantitative methylation percentages of 40 tumor suppressor genes $(53$ loci) in 53 primary breast tumors. The sidebars depict

not a confounder in methylation conversion. No significant association was found (for both analysis methods) between methylation of individual tumor suppressor genes and age at diagnosis.

\section{Prognostic value}

Of the primary tumor characteristics, lymph node positivity, ER or PR negativity (10\% cut-off for positivity), and HER2 positivity (DAKO score 3 ) were significantly correlated to worse survival (Table 2). When comparing survival curves of patients that showed methylation conversion from low to high or vice versa with those that did not, conversion of HLTF-2, ID4-2, SFRP4-1, and DAPK1 was correlated to worse overall survival (Fig. 5a). Conversion for these genes was entered in Cox proportional hazard analyses together, where SFRP4-1 (HR 2.3, $95 \%$ CI 1.03-5.05) and HLTF-2 (HR 2.2, $95 \%$ CI 1.09-4.56) remained significant (Table 3 ). When analyzing prognostic value of methylation status of the individual genes in the metastases for survival time from biopsy of metastases to end of follow-up, three out of the four aforementioned genes were again significant (ID4-2, SFRP4-1, and $D A P K 1$ ) (Fig. 5b). dissemination location, subtype (luminal A, luminal B, triple negative, and HER2 enriched), and ER status (according to $10 \%$ positivity)

\section{Correlation of methylation to mRNA expression by TCGA data extraction}

Despite possible heterogeneity in methylation between individual $\mathrm{CpG}$ sites within the same promoter region, we nevertheless tried to correlate methylation to mRNA expression by comparing the most closely located $\mathrm{CpG}$ sites between TCGA data and our MS-MLPA loci (criteria for matching: $<1000$ bp between $\mathrm{CpG}$ sites, significant inverse correlation, Pearson's $r>-0.2$; Online Resource Table 9). Note that these results thus need to be interpreted with caution.

The evaluated $\mathrm{CpG}$ sites/regions of $A T M, B C L 2, B R C A 1$, BRCA2, CACNA1G, CADM1, CASP8, CCND2, CD44, CDKN2B, CHFR1, DAPK1, ESR1, GSTP1, HLTF, ID4, MLH1, PRDM2, PTEN, RARB, RASSF1, RUNX3, TIMP3, TP73, and TWIST1 (15/40 genes) showed a significant inverse correlation with mRNA expression when quantitative data were used "Online Resource Table 6." Of these genes, fourteen showed higher methylation values in primaries compared to metastases in our cohort. For BNIP3, CACNA1A, CDH13, CDKN1B, FHIT, HIC1, SCGB3A1, SFRP4, SFRP5, and TGIFI (10/40 genes), no correlation was found between $\mathrm{CpG}$ site methylation and mRNA expression. 
Table 2 Cox proportional hazards modeling of tumor suppressor gene methylation

\begin{tabular}{|c|c|c|c|}
\hline \multirow[t]{2}{*}{ Predictor } & \multicolumn{2}{|l|}{ Bivariate model $p$ value } & \multirow[t]{2}{*}{$N$} \\
\hline & $\begin{array}{l}\text { Time between resection of } \\
\text { primary and end of follow-up }\end{array}$ & $\begin{array}{l}\text { Time between resection of } \\
\text { metastasis and end of follow-up }\end{array}$ & \\
\hline \multicolumn{4}{|c|}{ Methylation status in metastasis } \\
\hline ID4-2 & - & 0.009 & 53 \\
\hline SFRP4-1 & - & 0.023 & \\
\hline DAPK1 & - & 0.005 & \\
\hline DLC1-1 & - & 0.026 & \\
\hline GSTP & - & 0.035 & \\
\hline \multicolumn{4}{|c|}{ Conversion between primary and metastasis* } \\
\hline HLTF-2 & - & 0.023 & 53 \\
\hline ID4-2 & - & 0.025 & \\
\hline SFRP4-1 & - & 0.012 & \\
\hline DAPK1 & - & 0.041 & \\
\hline \multicolumn{4}{|l|}{ Molecular subtype } \\
\hline Luminal A & - & - & 10 \\
\hline Luminal B & 0.037 & 0.269 & 28 \\
\hline Triple negative & 0.047 & 0.187 & 12 \\
\hline HER2 enriched & 0.667 & 0.394 & 2 \\
\hline \multicolumn{4}{|l|}{ Location of metastasis } \\
\hline Brain & - & - & 10 \\
\hline Lung & 0.094 & 0.306 & 12 \\
\hline Liver & 0.754 & 0.812 & 10 \\
\hline Skin & 0.203 & 0.541 & 20 \\
\hline \multicolumn{4}{|l|}{ Tumor size } \\
\hline$<2 \mathrm{~cm}$ & - & - & 16 \\
\hline $2-5 \mathrm{~cm}$ & 0.699 & 0.039 & 25 \\
\hline$>5 \mathrm{~cm}$ & 0.756 & 0.330 & 6 \\
\hline \multicolumn{4}{|l|}{ Histologic type } \\
\hline Ductal & - & - & 44 \\
\hline Lobular & 0.553 & 0.940 & 4 \\
\hline Metaplastic & 0.774 & 0.823 & 3 \\
\hline Micropapillary & 0.506 & 0.344 & 1 \\
\hline \multicolumn{4}{|l|}{ Histologic grade } \\
\hline I & - & - & 1 \\
\hline II & 0.963 & 0.663 & 11 \\
\hline III & 0.026 & 0.303 & 40 \\
\hline MAI & 0.359 & 0.712 & 53 \\
\hline Lymph node status & 0.045 & 0.884 & 48 \\
\hline ER status & 0.000 & 0.260 & 53 \\
\hline PR status & 0.001 & 0.372 & 53 \\
\hline HER2 status & 0.041 & 0.613 & 53 \\
\hline Age at diagnosis primary & 0.385 & 0.202 & 53 \\
\hline Chemotherapy & 0.118 & 0.998 & 24 \\
\hline Radiotherapy & 0.064 & 0.024 & 37 \\
\hline Hormone therapy & 0.236 & 0.907 & 22 \\
\hline \multicolumn{4}{|l|}{ Combination therapy } \\
\hline Chemoradiation & - & - & 7 \\
\hline Radiohormonal therapy & 0.097 & 0.822 & 3 \\
\hline
\end{tabular}


Table 2 continued

\begin{tabular}{|c|c|c|c|}
\hline \multirow[t]{2}{*}{ Predictor } & \multicolumn{2}{|l|}{ Bivariate model $p$ value } & \multirow[t]{2}{*}{$N$} \\
\hline & $\begin{array}{l}\text { Time between resection of } \\
\text { primary and end of follow-up }\end{array}$ & $\begin{array}{l}\text { Time between resection of } \\
\text { metastasis and end of follow-up }\end{array}$ & \\
\hline Chemohormonal therapy & 0.114 & 0.992 & 4 \\
\hline Chemoradiation + hormonal therapy & 0.931 & 0.174 & 4 \\
\hline CMI primaries & 0.642 & 0.876 & 53 \\
\hline CMI metastases & 0.726 & 0.630 & 53 \\
\hline
\end{tabular}

Clinicopathological characteristics are compared to time between resection of primary or metastasis and end of follow-up

* Variables which are put in the multivariate model

Table 3 Multivariate model of conversion between primary and metastasis in time between resection of metastasis and end of followup

\begin{tabular}{lllll}
\hline Parameter & Significance & Hazard ratio & $95 \%$ CI & \\
\cline { 3 - 5 } & & & Lower limit & Upper limit \\
\hline SFRP4-1 & 0.042 & 2.279 & 1.028 & 5.052 \\
HLTF-2 & 0.029 & 2.223 & 1.085 & 4.556
\end{tabular}

Bivariate analysis identified several significant $(p<0.05)$ predictors of survival. Reference categories were set for those predictors with more than two categories. Based upon number of patients included, 4 predictors could be tested in multivariate Cox proportional hazard models. HLTF-2, ID4-2, SFRP4-1, and DAPK1 were used to generate composite models through forward conditional testing, with $p<0.05$ as the basis for retaining and removing variables

\section{Discussion}

DNA methylation has a similar potential as genetic alterations in serving as a selectable driver during clonal expansion or metastatic dissemination and could therefore yield valuable markers for cancer detection and prognosis as well as targets for new therapeutic strategies [20]. Our study design allowed comparison of primary breast tumors to their paired distant metastases at different locations, enabling intra- and inter-individual comparison.

Our results show a general tendency for lower methylation at primary tumor-methylated regions in the matched metastases of 21/40 tumor suppressor genes. It is unlikely that admixture of cells from the tumor micro-environment at distant sites have caused these lower methylation values. First, we only included metastatic samples that contained at least $80 \%$ tumor. Second, methylation values in normal breast were lower than in normal tissues from skin, lung, brain, and liver, so admixture of such normal cells (especially from liver and brain) would have raised methylation values. Third, all normal tissues clustered together in unsupervised analysis, which also showed that primary tumors and their paired metastases cluster together. Therefore, most of these hypermethylation events are likely patient specific and subject to specific selection across metastatic dissemination and expansion, emphasizing the need for personalized cancer treatment.

Higher CMI correlated with higher MAI as did methylation values of individual genes, indicating that proliferation rate correlates with methylation, which is biologically plausible. Adjuvant chemotherapy or hormonal therapy did not seem to influence methylation conversion.

To our knowledge, our study is the first that compared promoter methylation in a large group of multiple localizations of distant human breast cancer metastases to their matched primary breast carcinomas and we tried to apply the "reporting recommendations for tumor markers" (REMARK criteria) as adequately as possible [21]. Several studies have been performed addressing methylation differences between primary tumors and metastases. However, their methods failed to draw conclusions on intrapatient differences and site-specific markers. Limitations included: description of a single metastatic site or tumor suppressor gene, non-matched pairs of primaries and metastases, methylation only in the primary tumor (compared to the metastasizing tendency), or the use of mouse models instead of patient material [11, 12, 22-26]. Rivenbark et al. demonstrated "epigenetic progression" by showing more methylation in lymph node metastases compared to the primary breast tumor [13], but $\mathrm{Wu}$ et al. showed no differences in methylation of seven tumor suppressor genes in primary breast carcinomas compared to their matched distant metastases [27]. The discrepant findings with our generally lower methylation values in distant metastases (largely in line with results in head and neck squamous cell carcinomas [28]) are likely related to differences in distant metastasis localizations, differences in study populations and sample sizes, pairing of normal tissue, the inclusion of paired metastases, and variation in tumor suppressor genes and $\mathrm{CpG}$ regions studied, Further, methodologies for demonstration of methylation status (QM-MSP, methylation-specific PCR analysis, bisulfite sequencing, differential methylation hybridization, etc.) 

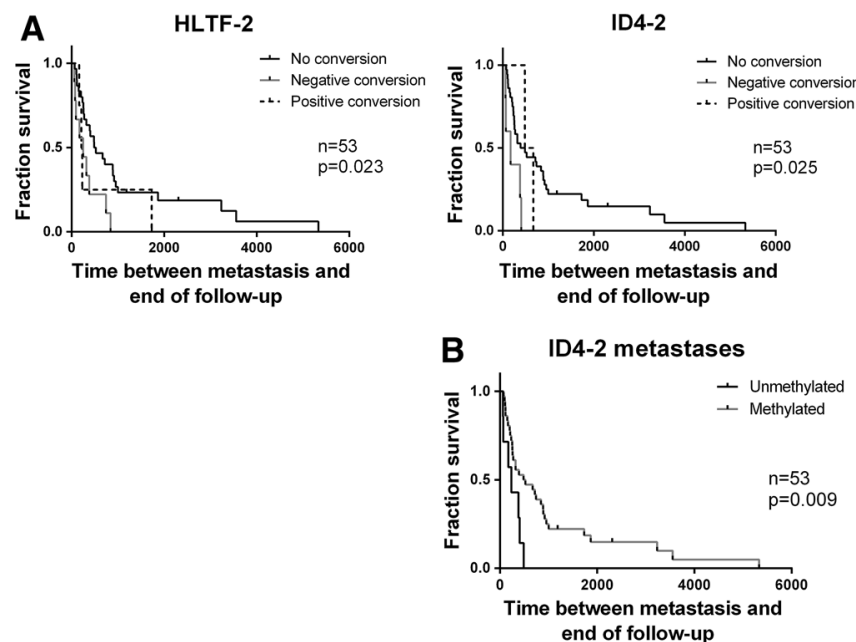

Fig. 5 Kaplan-Meier survival curves of time between resection of metastasis to end of follow-up of HLTF-2, ID4-2, SFRP4-1 and $D A P K 1$ of conversion of methylation status in the primary tumors compared to paired metastases (a). The dashed line depicts conversion from negative in the primary tumor to positive in the metastasis and the gray line depicts conversion from positive in the primary tumor to negative in the metastasis. Survival curves of ID4-2, SFRP41, and DAPK1 of methylation status of metastases are shown in (b). Chromosome location HLTF-2: chr3:148747904-148804341, CpG

differ between studies. In our institute, we have extensive experience using MS-MLPA [10, 29-31], a restriction enzyme-based assay that allows a multi-target approach on small amounts of DNA extracted from formalin-fixed paraffin embedded material. This technique shows a very good correlation with other techniques such as bisulfite pyrosequencing and (QM) MSP [32-37]. Besides, a tumor or metastasis-initiating clone or sub-clone in each individual has a unique DNA methylation signature that is closely maintained across metastatic dissemination [20]. However, for each tumor, we chose one of many available tissue blocks (that contained the largest amount of tumor load), which could have led to sampling bias. A previous study from our group clearly demonstrated that, although most variation in methylation status is present between individual breast cancers, clonal epigenetic heterogeneity is seen within most primary breast carcinomas, indicating that methylation results from a single random sample may not be representative of the whole tumor [30]. In addition, for 12 genes, two different CpG loci were analyzed separately, and exact results showed differences in methylation frequencies, indicating the presence of heterogeneous methylation. However, unsupervised hierarchical clustering showed an almost perfect correlation between six and eight of the 12 genes of which different $\mathrm{CpG}$ sites were analyzed. These limitations could explain perhaps some but clearly not all of the differences in methylation values between primary and metastasis.

To correct for the differences between locations of dissemination, differences between molecular subtypes should be
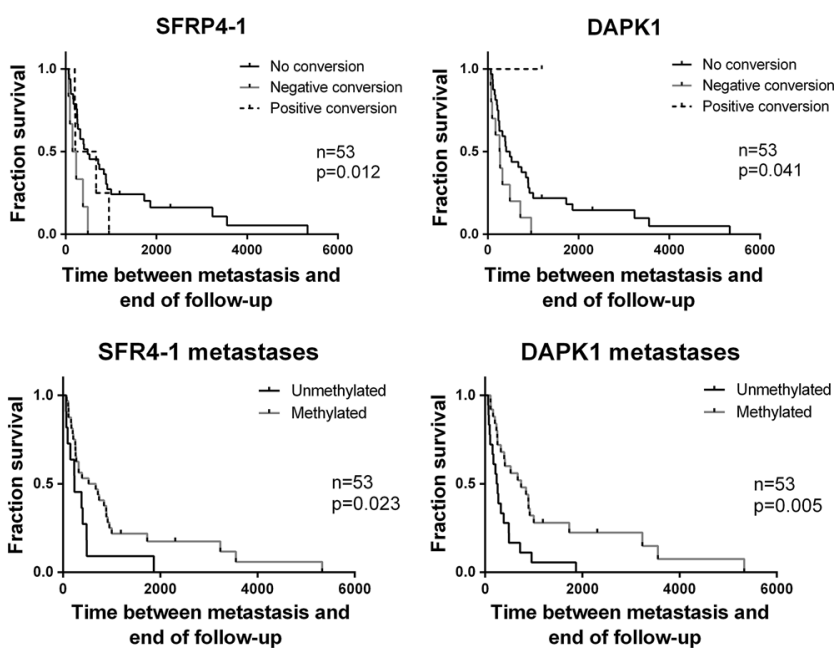

site MS-MLPA probe: 148804223 , \#bp from probe to TSS: -105 and from probe to ATG: -105. Chromosome location ID4-2: chr6:19837601-19842431, CpG site MS-MLPA probe: 19837620, \#bp from probe to TSS: -20 and from probe to ATG: 365 . Chromosome location SFRP4-1: chr7:37945535-37956525, CpG site MS-MLPA probe: 37956166 , \#bp from probe to TSS: -10632 and from probe to ATG: -9086. Chromosome location DAPK1: chr9:90113885-90323549, CpG site MS-MLPA probe: 90113281, \#bp from probe to TSS: 603 and from probe to ATG: 711

taken into account, since they are known to preferentially metastasize to specific distant sites [4, 38]. For instance, a general hypomethylation of basal-like tumors compared to differential methylation across non-basal-like subtypes is often reported $[38,39]$. We indeed saw some clustering of triple negative tumors and one cluster almost entirely composed of ER-positive cancers, but no evident hypomethylation was seen compared to other subtypes. Distinct methylation patterns relative to breast cancer subtype and normal breast tissue as shown by Bardowell et al. [38] were also not seen. Further, some of the chosen genes were significantly more methylated in tumors that metastasized to specific localizations (even when corrected for molecular subtype), which could lead to novel biomarkers predicting site of distant metastases and adjuvant targeted therapy strategies that could prevent such metastases from becoming clinically manifest.

In a therapeutic setting, the correlation between methylation and mRNA/protein expression may become relevant, which is why we explored TCGA data. Generally, methylation at the investigated CpG sites by MS-MLPA, seemed inversely correlated to mRNA expression levels as demonstrated before [40] (despite possible heterogeneity in methylation between individual $\mathrm{CpG}$ sites used for MSMLPA and TCGA test), indicating their relevance in gene silencing. Future studies should take into account actual protein expression of tumor suppressor genes in metastases in relation to methylation status.

Theoretically, less methylation in metastases would prognostically be beneficial for the patient because of 
reactivation of these tumor suppressor genes. However, survival analysis showed that conversion of HLTF-2, ID42, SFRP4-1, and DAPK1 from positive in the primary tumor to negative in the metastasis was correlated to worse overall survival. Interestingly, methylation status of 3/4 of these genes (ID4-2, SFRP4-1 and DAPK1) predicted worse survival when hypermethylated in metastases. Most important independent predictors for shorter survival time over lymph node positivity and ER status were SFRP4 and HLTF, which are known predictors of worse survival. Hypermethylation of HLTF seems to predict poor outcome in colorectal [41, 42] and lung cancer [43]. SFRP4 is been shown to be an independent predictor of shorter survival in myelodysplastic syndrome [44] and invasive bladder cancer [45]. However, these studies emphasize hypermethylation status in primary tumors, and no studies were found on hypermethylation of these markers in paired metastases in relation to survival. Promoter hypermethylation of tumor suppressor genes is known to be an early event during carcinogenesis $[9,10]$. There are several possible explanations for the trend that less promoter methylation of the investigated genes is seen in the metastases. First, the spread of tumor cells may take place even prior to methylation. It has been demonstrated before that in breast, prostate, and esophageal cancer, bone marrow disseminated tumor cells (DTCs: any tumor cell that has left the primary lesion and traveled to an ectopic environment, not necessarily forming a metastasis) display significantly fewer genetic aberrations than primary tumor cells [46-49]. Dissemination of tumor cells that are still evolving may lead to allopatric selection and expansion of variant cells adapted to specific microenvironments [50]. Second, it could be that methylation is a dynamic process and may even vary in different stages of the cell cycle. Graff et al. have shown that E-cadherin (a gene involved in homotypic cell-cell adhesion) in cell lines is hypermethylated when put in a culture model system for basement membrane invasion and hypomethylated in a tumor growth model [51]. The reversibility of methylation of tumor suppressor genes could therefore be beneficial to tumor spread, whether it is a random process or a response to specific signals.

In summary, we have shown that hypermethylation of tumor suppressor genes detected by MS-MLPA is generally lower in the distant metastases compared to the primary tumors. We already knew that hypermethylation, in contrast to DNA mutations, is reversible, but whether this is a random or controlled principle has not been fully elucidated. The question rises if the difference in methylation pattern between these primaries and metastases could be explained by the loss/rearrangement of hypermethylation. Since we have shown that the $21 / 40$ tested tumor suppressor genes show less methylation in metastases with respect to their matched primary carcinomas, methylation is probably not an epigenetic factor that could be used for therapy against metastatic tumor spread. However, since different metastasizing localizations show different methylation patterns, screening for a specific pattern that predicts most likely site of metastases could be a useful clinical tool. Further, methylation status of several genes seems to predict survival after metastases. Therefore, more tumor suppressor genes should be screened on larger databases and heterogeneity should be ruled out to include all tumor subclones.

Acknowledgments This study is supported by the Dutch Cancer Society Grant UU 2011-5195 and Philips Consumer Lifestyle. We especially would like to thank the University Medical Center Utrecht, the Meander Medical Center Amersfoort, the Deventer Hospital, the Rijnstate Hospital Arnhem, Tergooi Hospitals, the Academic Medical Center Amsterdam, the Radboud University Nijmegen Medical Center, the Canisius Wilhelmina Hospital Nijmegen, the Netherlands Cancer Institute Amsterdam, the Medical Center Alkmaar, the Medical Center Zaandam, the University Medical Center Groningen, the St. Antonius Hospital Nieuwegein, the Diakonessenhuis Utrecht, the Free University Medical Center Amsterdam, the Erasmus Medical Center Rotterdam, the Gelre hospital Apeldoorn, the Isala clinics Zwolle, the Laboratory for Pathology Enschede, the Laboratory for Pathology Dordrecht, and the Laboratory for Pathology Foundation Sazinon Hoogeveen for providing archival tissue for this study.

Conflict of interest The authors declare that they have no conflict of interest.

Ethical standards This study was performed in accordance with the institutional medical ethical guidelines. The use of anonymous or coded left over material for scientific purposes is part of the standard treatment agreement with patients, and therefore, informed consent was not required according to Dutch law [17].

Funding This work was supported by the Dutch Cancer Society Grant UU 2011-5195 and Philips Consumer Lifestyle.

Open Access This article is distributed under the terms of the Creative Commons Attribution-NonCommercial 4.0 International License (http://creativecommons.org/licenses/by-nc/4.0/), which permits any noncommercial use, distribution, and reproduction in any medium, provided you give appropriate credit to the original author(s) and the source, provide a link to the Creative Commons license, and indicate if changes were made.

\section{References}

1. WHO, international agency for research on cancer (2013) Latest world cancer statistics. Global cancer burden rises to 14.1 million new cases in 2012: marked increase in breast cancers must be addressed. World health organization, Geneva

2. van den Hurk CJ, Eckel R, van de Poll-Franse LV et al (2011) Unfavourable pattern of metastases in M0 breast cancer patients during 1978-2008: a population-based analysis of the Munich Cancer Registry. Breast Cancer Res Treat 128:795-805. doi:10. 1007/s10549-011-1372-y 
3. Ferlicot S, Vincent-Salomon A, Medioni J et al (2004) Wide metastatic spreading in infiltrating lobular carcinoma of the breast. Eur J Cancer 40:336-341. doi:10.1016/j.ejca.2003.08.007

4. Kennecke H, Yerushalmi R, Woods R et al (2010) Metastatic behavior of breast cancer subtypes. J Clin Oncol 28:3271-3277. doi:10.1200/JCO.2009.25.9820

5. Tseng LM, Hsu NC, Chen SC et al (2013) Distant metastasis in triple-negative breast cancer. Neoplasma 60:290-294. doi:10. 4149/neo_2013_038

6. Paget S (1989) The distribution of secondary growths in cancer of the breast. 1889. Cancer Metastasis Rev 8:98-101

7. Connolly R, Stearns V (2012) Epigenetics as a therapeutic target in breast cancer. J Mammary Gland Biol Neoplasia 17:191-204. doi:10.1007/s10911-012-9263-3

8. Lorusso G, Ruegg C (2012) New insights into the mechanisms of organ-specific breast cancer metastasis. Semin Cancer Biol 22:226-233. doi:10.1016/j.semcancer.2012.03.007

9. Park SY, Kwon HJ, Lee HE et al (2011) Promoter CpG island hypermethylation during breast cancer progression. Virchows Arch 458:73-84. doi:10.1007/s00428-010-1013-6

10. Verschuur-Maes AH, de Bruin PC, van Diest PJ (2012) Epigenetic progression of columnar cell lesions of the breast to invasive breast cancer. Breast Cancer Res Treat 136:705-715. doi:10. 1007/s10549-012-2301-4

11. Chen L, Zhu Z, Sun X et al (2009) Down-regulation of tumor suppressor gene FEZ1/LZTS1 in breast carcinoma involves promoter methylation and associates with metastasis. Breast Cancer Res Treat 116:471-478. doi:10.1007/s10549-008-0147-6

12. Swift-Scanlan T, Vang R, Blackford A et al (2011) Methylated genes in breast cancer: associations with clinical and histopathological features in a familial breast cancer cohort. Cancer Biol Ther 11:853-865. doi:10.4161/cbt.11.10.15177

13. Rivenbark AG, Livasy CA, Boyd CE et al (2007) Methylationdependent silencing of CST6 in primary human breast tumors and metastatic lesions. Exp Mol Pathol 83:188-197. doi:10.1016/j. yexmp.2007.03.008

14. Hoefnagel LD, van de Vijver MJ, van Slooten HJ et al (2010) Receptor conversion in distant breast cancer metastases. Breast Cancer Res 12:R75. doi:10.1186/bcr2645

15. Hoefnagel LD, Moelans CB, Meijer SL et al (2012) Prognostic value of estrogen receptor alpha and progesterone receptor conversion in distant breast cancer metastases. Cancer 118:4929-4935. doi:10. $1002 /$ cncr. 27518

16. Hoefnagel LD, van der Groep P, van de Vijver MJ et al (2013) Discordance in ERalpha, PR and HER2 receptor status across different distant breast cancer metastases within the same patient. Ann Oncol 24:3017-3023. doi:10.1093/annonc/mdt390

17. van Diest PJ (2002) No consent should be needed for using leftover body material for scientific purposes. For BMJ 325:648-651

18. Cerami E, Gao J, Dogrusoz U et al (2012) The cBio cancer genomics portal: an open platform for exploring multidimensional cancer genomics data. Cancer Discov 2:401-404. doi:10. 1158/2159-8290.CD-12-0095

19. Gao J, Aksoy BA, Dogrusoz U et al (2013) Integrative analysis of complex cancer genomics and clinical profiles using the cBioPortal. Sci Signal 6(269):pl1. doi:10.1126/scisignal.2004088

20. Aryee MJ, Liu W, Engelmann JC et al (2013) DNA methylation alterations exhibit intra individual stability and interindividual heterogeneity in prostate cancer metastases. Sci Transl Med 5:169ra10. doi:10.1126/scitranslmed.3005211

21. McShane LM, Altman DG, Sauerbrei W et al (2006) REporting recommendations for tumor MARKer prognostic studies (REMARK). Breast Cancer Res Treat 100:229-235. doi:10.1007/ s10549-006-9242-8

22. Acosta D, Suzuki M, Connolly D et al (2011) DNA methylation changes in murine breast adenocarcinomas allow the identification of candidate genes for human breast carcinogenesis. Mamm Genome 22:249-259. doi:10.1007/s00335-011-9318-6

23. Noetzel E, Rose M, Sevinc E et al (2010) Intermediate filament dynamics and breast cancer: aberrant promoter methylation of the Synemin gene is associated with early tumor relapse. Oncogene 29:4814-4825. doi:10.1038/onc.2010.229

24. Salhia B, Kiefer J, Ross JT et al (2014) Integrated genomic and epigenomic analysis of breast cancer brain metastasis. PLoS One 9:e85448. doi:10.1371/journal.pone.0085448

25. Carraway HE, Wang S, Blackford A et al (2009) Promoter hypermethylation in sentinel lymph nodes as a marker for breast cancer recurrence. Breast Cancer Res Treat 114:315-325. doi:10. 1007/s10549-008-0004-7

26. Mehrotra J, Vali M, McVeigh M et al (2004) Very high frequency of hypermethylated genes in breast cancer metastasis to the bone, brain, and lung. Clin Cancer Res 10:3104-3109

27. Wu JM, Fackler MJ, Halushka MK et al (2008) Heterogeneity of breast cancer metastases: comparison of therapeutic target expression and promoter methylation between primary tumors and their multifocal metastases. Clin Cancer Res 14:1938-1946. doi:10.1158/1078-0432.CCR-07-4082

28. Smiraglia DJ, Smith LT, Lang JC et al (2003) Differential targets of $\mathrm{CpG}$ island hypermethylation in primary and metastatic head and neck squamous cell carcinoma (HNSCC). J Med Genet 40:25-33

29. Moelans CB, Verschuur-Maes AH, van Diest PJ (2011) Frequent promoter hypermethylation of BRCA2, CDH13, MSH6, PAX5, PAX6 and WT1 in ductal carcinoma in situ and invasive breast cancer. J Pathol 225:222-231. doi:10.1002/path.2930

30. Moelans CB, de Groot JS, Pan X et al (2014) Clonal intratumor heterogeneity of promoter hypermethylation in breast cancer by MS-MLPA. Mod Pathol 27:869-874. doi:10.1038/modpathol. 2013.207

31. Suijkerbuijk KP, Fackler MJ, Sukumar S et al (2008) Methylation is less abundant in BRCA1-associated compared with sporadic breast cancer. Ann Oncol 19:1870-1874. doi:10.1093/annonc/ $\operatorname{mdn} 409$

32. Suijkerbuijk KP, Pan X, van der Wall E et al (2010) Comparison of different promoter methylation assays in breast cancer. Anal Cell Pathol (Amst) 33:133-141. doi:10.3233/ACP-CLO-20100542

33. Leong KJ, Wei W, Tannahill LA et al (2011) Methylation profiling of rectal cancer identifies novel markers of early-stage disease. Br J Surg 98:724-734. doi:10.1002/bjs.7422

34. Cardoso LC, Tenorio Castano JA, Pereira HS et al (2012) Constitutional and somatic methylation status of DMRH19 and KvDMR in Wilms tumor patients. Genet Mol Biol 35:714-724. doi:10.1590/S1415-47572012005000073

35. Lopez F, Sampedro T, Llorente JL et al (2014) Utility of MSMLPA in DNA methylation profiling in primary laryngeal squamous cell carcinoma. Oral Oncol 50:291-297. doi:10.1016/j. oraloncology.2014.01.003

36. Furlan D, Sahnane N, Mazzoni M et al (2013) Diagnostic utility of MS-MLPA in DNA methylation profiling of adenocarcinomas and neuroendocrine carcinomas of the colon-rectum. Virchows Arch 462:47-56. doi:10.1007/s00428-012-1348-2

37. Pineda M, Mur P, Iniesta MD et al (2012) MLH1 methylation screening is effective in identifying epimutation carriers. Eur $\mathrm{J}$ Hum Genet 20:1256-1264. doi:10.1038/ejhg.2012.136

38. Bardowell SA, Parker J, Fan C et al (2013) Differential methylation relative to breast cancer subtype and matched normal tissue reveals distinct patterns. Breast Cancer Res Treat 142:365-380. doi:10.1007/s10549-013-2738-0

39. Ulirsch J, Fan C, Knafl G et al (2013) Vimentin DNA methylation predicts survival in breast cancer. Breast Cancer Res Treat 137:383-396. doi:10.1007/s10549-012-2353-5 
40. Wang D, Yang PN, Chen J et al (2014) Promoter hypermethylation may be an important mechanism of the transcriptional inactivation of ARRDC3, GATA5, and ELP3 in invasive ductal breast carcinoma. Mol Cell Biochem 396:67-77. doi:10.1007/ s11010-014-2143-y

41. Philipp AB, Nagel D, Stieber P et al (2014) Circulating cell-free methylated DNA and lactate dehydrogenase release in colorectal cancer. BMC Cancer 14(1):245. doi:10.1186/1471-2407-14-245

42. Philipp AB, Stieber P, Nagel D et al (2012) Prognostic role of methylated free circulating DNA in colorectal cancer. Int $\mathbf{J}$ Cancer 131:2308-2319. doi:10.1002/ijc.27505

43. Castro M, Grau L, Puerta P et al (2010) Multiplexed methylation profiles of tumor suppressor genes and clinical outcome in lung cancer. J Transl Med 8(86):1479-5876. doi:10.1186/1479-58768-86

44. Wang H, Fan R, Wang XQ et al (2013) Methylation of Wnt antagonist genes: a useful prognostic marker for myelodysplastic syndrome. Ann Hematol 92:199-209. doi:10.1007/s00277-0121595-y

45. Marsit CJ, Karagas MR, Andrew A et al (2005) Epigenetic inactivation of SFRP genes and TP53 alteration act jointly as markers of invasive bladder cancer. Cancer Res 65:7081-7085. doi:10.1158/0008-5472.can-05-0267
46. Schardt JA, Meyer M, Hartmann $\mathrm{CH}$ et al (2005) Genomic analysis of single cytokeratin-positive cells from bone marrow reveals early mutational events in breast cancer. Cancer Cell 8:227-239. doi:10.1016/j.ccr.2005.08.003

47. Schmidt-Kittler O, Ragg T, Daskalakis A et al (2003) From latent disseminated cells to overt metastasis: genetic analysis of systemic breast cancer progression. Proc Natl Acad Sci USA 100:7737-7742. doi:10.1073/pnas.1331931100

48. Stoecklein NH, Hosch SB, Bezler M et al (2008) Direct genetic analysis of single disseminated cancer cells for prediction of outcome and therapy selection in esophageal cancer. Cancer Cell 13:441-453. doi:10.1016/j.ccr.2008.04.005

49. Weckermann D, Polzer B, Ragg T et al (2009) Perioperative activation of disseminated tumor cells in bone marrow of patients with prostate cancer. J Clin Oncol 27:1549-1556. doi:10.1200/ JCO.2008.17.0563

50. Klein CA (2009) Parallel progression of primary tumours and metastases. Nat Rev Cancer 9:302-312. doi:10.1038/nrc2627

51. Graff JR, Gabrielson E, Fujii $\mathrm{H}$ et al (2000) Methylation patterns of the E-cadherin 5' CpG island are unstable and reflect the dynamic, heterogeneous loss of E-cadherin expression during metastatic progression. J Biol Chem 275:2727-2732 